Araştırma Makalesi - Research Article

\title{
Uyarılmış Spin-3/2 Nötrinoların Foton İndüklü Reaksiyonda Doğrudan Olmayan Üretimi
}

\author{
Volkan ÇETINNKAYA ${ }^{1 *}$
}

Revize / Revised: 08/12/2019

Kabul / Accepted: 09/12/2019

\section{$\overline{\mathbf{O Z Z}}$}

$\mathrm{Bu}$ çalışmada, $\mathrm{e}^{-} \gamma \rightarrow \mathrm{v}_{\mathrm{e}} \mathrm{W}^{-}$sürecinde uyarılmış nötrinonun t-kanalında doğrudan olmayan üretimi araştırılmıştır. Burada e $\mathrm{e}^{-} \gamma \rightarrow \mathrm{ve}_{\mathrm{e}} \mathrm{W}^{-}$süreci, $\mathrm{e}^{-} \mathrm{e}^{+}$(CLIC) çarpıştırıcısının bir alt süreci olarak ele alınmıştır. $\mathrm{e}^{-} \mathrm{e}^{+}$sisteminin kütle merkezi enerjisi $3000 \mathrm{GeV}$ ve yıllık 1şınlık değeri $5000 \mathrm{fb}^{-1}$ alınmıştır. Ancak Compton geri saçılan foton dağılımından dolayı e $\gamma$ çarpışmasının kütle merkezi enerjisi $2733 \mathrm{GeV}$ olmuştur. Tüm hesaplamalarda kompozitlik ölçeği, uyarılmış nötrino kütlesine eşit kabul edilmiştir. Uyarılmış nötrinolar, Standart Model (SM) leptonları ve ayar bozonları ( $\gamma, \mathrm{W}$ ve Z) arasındaki etkileşmeler için efektif akımlar kullanılmıştır. Buna göre sinyal ve aynı son durumu veren ardalanlar için tesir kesiti değerleri elde edilmiştir. Sinyal ve ardalan değerleri arasındaki farklılık SM lagranjiyenine eklenen efektif etkileşme terimlerinden kaynaklanmıştır. Deneysel gözlenebilirliğin saptanması için istatistik yöntemler kullanılabilir. Burada yöntem olarak istatistik önem (SS) kullanılmıştır. SS $\geq 3$ kriterine göre uyarılmış spin-3/2 nötrinoların $3000 \mathrm{GeV}$ kütle değerine kadar (bu değer de dâhil) $\mathrm{J}_{1}$ ve $\mathrm{J}_{2}$ akımı için \%95 güvenirlilik seviyesinde gözlenebileceği sonucuna varılmıştır. İstatistik yöntem kullanılırken son durumdaki W'nun hadronik bozunduğu varsayılmıştır. Bu çalışmada, ayrıca, $\mathrm{J}_{2}$ akımı kullanılarak serbest bağlaşım parametrelerinin iki-boyutlu kontur grafikleri elde edilmiştir. Buna göre bu parametreler, uyarılmış nötrinoların daha küçük kütle değerlerinde, beklendiği gibi, daha küçük bölgeye sınırlandırılmışlardır. Son olarak,

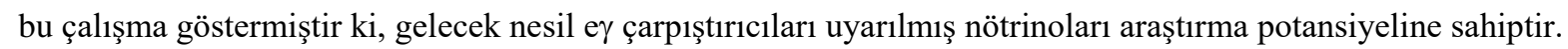

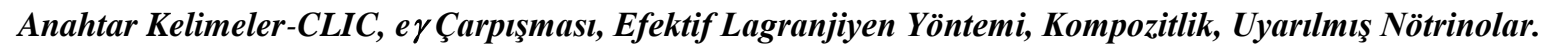

1*Sorumlu yazar iletişim: volkan.cetinkaya@dpu.edu.tr (https://orcid.org/0000-0001-7056-3325)

Fizik Bölümü, Kütahya Dumlupınar Üniversitesi, Kütahya 


\title{
Indirect Production of Excited Spin-3/2 Neutrinos in Photon Induced Reaction
}

\begin{abstract}
In this study, we investigate the indirect production of the excited neutrino in the $\mathrm{t}$-channel in the $\mathrm{e}^{-} \gamma \rightarrow \mathrm{v}_{\mathrm{e}} \mathrm{W}^{-}$ process. Here, the process $\mathrm{e}^{-} \gamma \rightarrow \mathrm{v}_{\mathrm{e}} \mathrm{W}^{-}$is considered as a subprocess of the $\mathrm{e}^{-} \mathrm{e}^{+}$(CLIC) collider. The center-ofmass energy and the integrated luminosity of the $\mathrm{e}^{-} \mathrm{e}^{+}$system are taken as $3000 \mathrm{GeV}$ and $5000 \mathrm{fb}^{-1}$, respectively. However, due to the Compton backscattering photon distribution, the center-of-mass energy of the $\mathrm{e}^{-} \gamma$ collision is $2733 \mathrm{GeV}$. In all calculations, the compositeness scale is assumed to be equal to the excited neutrino mass. Effective currents are used for interactions among excited neutrinos, the Standard Model (SM) leptons and gauge bosons $(\gamma, \mathrm{W}$ and $\mathrm{Z}$ ). Accordingly, the cross-section values have been obtained for the signal and the backgrounds giving the same final state. The difference between the signal and the background values is due to the effective interaction terms added to the SM lagrangian. Statistical methods can be used to determine experimental observability. Statistical Significance (SS) is used as a method here. It is concluded that the excited spin-3/2 neutrinos for the criteria $S S \geq 3$ can be observed at $95 \%$ confidence level for $J_{1}$ and $J_{2}$ currents up to $3000 \mathrm{GeV}$ mass value (including this value). When using the statistical method, it is assumed that $\mathrm{W}$ in the final state decays hadronically. In this study, we have obtained two-dimensional contour graphs of free coupling parameters by using $\mathbf{J}_{2}$ current. Accordingly, these parameters are limited to the smaller region, as expected, at smaller mass values of the excited neutrinos. Finally, this study has shown that the next generation ey colliders have the potential to investigate the excited neutrinos.
\end{abstract}

Keywords- CLIC, Compositeness, e $\gamma$ Collisions, Effective Lagrangian Method, Excited Neutrinos. 


\section{GİRIŞ}

Parçacık fiziğinin Standart Modeli (SM), temel parçacıkları ve onların birbirleriyle olan etkileşmelerini elektrozayıf ölçeğe kadar başarılı bir biçimde açıklamaktadır. SM'nin pek çok öngörüsü bugüne kadar yapılan deneylerle oldukça uyum içindedir. Bunun yanında, SM ile açıklanamayan problemler halen çözüm beklemeye devam etmektedir. Örneğin, yalnızca deneyle belirlenebilen, temel parçacıkların kütleleri de dâhil, yirmi kadar parametre bu modelle öngörülememektedir. Buna ek olarak kütleçekim kuramı halen bu modele dâhil edilememiştir. Ayrıca, karanlık madde, karanlık enerji olguları ve madde-karşıtmadde asimetrisi de SM ile açıklanamamaktadır. Bu problemlere çözüm önerisi getiren çeşitli SM ötesi modeller kurulmuş ve bazıları yüksek enerjili çarpıştırıcılarda sınanmıştır. Ancak bugüne kadar SM etkileşmeleri ile uyumsuz bir sinyale rastlanmamıştır. Bununla birlikte, kuark ve leptonların bilinen üç ailesi vardır ve fermiyon ailelerinin neden birbirini tekrarladığı SM tarafından açıklanamamaktadır. Bu problemi çözmek amacıyla SM ötesi bir model olan kompozitlik ortaya atılmıştır [1-3]. Bu model, tüm fermiyonların daha temel madde bileşenleri olan preonlardan oluştuğunu söyler. Bu parçacıkların var olduğuna ilişkin bir kanıt, kompozitlik adı verilen bir enerji ölçeğinde, $\Lambda$, yeni etkileşmelerin deneysel olarak gözlenmesiyle olacaktır. Uyarılmış durumların ortaya çıkması, bilinen fermiyonların kompozit yapısının doğal bir sonucu olacaktır [4-7]. Böylece leptonların kompozit oldukları varsayılırsa, uyarılmış spin-1/2 leptonlar, üç adet spin-1/2 alt parçacığın [1] ya da spin-1/2 ve spin-0 alt parçacıkların [2] bağlı durumu olarak yazılabilir. Benzer şekilde, kompozit modeller çerçevesinde [8], spin-3/2 lepton bağlı durumlarının da üç adet spin-1/2 alt parçacık [1-3] ile ya da spin-1/2 ve spin-1 alt parçacıklar ile kurulması mümkündür.

Uyarılmış spin-1/2 leptonlar, hadron-hadron, lepton-lepton, lepton-hadron çarpıştırıcılarında ve foton indüklü reaksiyonlarda incelenmiştir [9-29]. Aynı zamanda, uyarılmış spin-3/2 leptonlar da çeşitli çarpışmalarda ele alınmıştır [30-35].

Uyarılmış spin-1/2 nötrinolar için kütle limiti L3 İşbirliği tarafından, $\mathrm{e}^{+} \mathrm{e}^{-}$çarpıştırıcısında bu nötrinoların çift üretimiyle, $m^{*}>102,6 \mathrm{GeV}$ olarak belirlenmiştir [36]. H1 İşbirliği tarafından bu değer daha da artırılmış ve ep çarpıştırıcısındaki tek üretime bakılarak \%95 güvenirlilik seviyesinde $\mathrm{m}^{*}>213 \mathrm{GeV}$ olmuştur [37]. Son zamanlarda ATLAS İşbirliği uyarılmış nötrinoların 1,6 TeV'den daha büyük kütleye sahip olmaları gerektiğini açıklamıştır [38]. Bu nötrinolara her ne kadar kütle limiti getirilmişse de uyarılmış spin-3/2 nötrinolar için literatürde henüz böyle bir çalışma yoktur. Bu yüzden spin-3/2 nötrinolar ile yapılan hesaplamalarda da spin-1/2 için getirilen kütle sınırlamalarının dikkate alınması akla yatkın gelmektedir.

Bu çalışma, lepton bazlı bir foton indüklü reaksiyonda, uyarılmış spin-3/2 nötrinonun doğrudan olmayan üretimini içermektedir. Çalışmanın içeriği şu şekildedir: Bölüm II'de uyarılmış nötrinolar için fenomenolojik akımlar ve bozunma genişliklerinin yer aldığı teorik çerçeve ele alınacaktır. Bölüm III'te e $\gamma$ çarpışmasında doğrudan olmayan üretim için sinyal tesir kesitleri ve karşılık gelen ardalan değerleri hesaplanmıştır. Burada, yüksek kütle merkezi enerjili ve yüksek 1şınlıklı gelecek nesil $\mathrm{e}^{-} \mathrm{e}^{+}$çarpıştırıcısı olan CLIC (Kompakt Lineer Çarpıştırıcı)' in [39-43] e $\gamma$ modu dikkate alınmıştır. Foton dağılımı olarak Compton geri saçılan foton (lazer foton) dağılımı kullanılmıştır. $\mathrm{e}^{-} \mathrm{e}^{+}$sisteminin $3000 \mathrm{GeV}$ olan kütle merkezi enerjisi fotonun pozitron enerjisinin maksimum \%83'ünü almasından dolayı $2733 \mathrm{GeV}$ olarak alınmıştır. Son olarak uyarılmış spin-3/2 nötrino bağlaşımlarının limitlerini veren iki-boyutlu kontur grafikleri elde edilmiştir. Çalışmanın sonuçları (Bölüm IV) göstermiştir ki foton indüklü reaksiyonlar uyarılmış nötrinoların araştırılması için önemli bir potansiyele sahiptir.

\section{I.TEORIKK ÇERÇEVE}

Bilindiği gibi parçacık fiziğinin Standart Modeli, günümüze kadar yapılan deneylerle uyum içinde kalarak doğayı elektrozayıf ölçekte tasvir etme başarısını göstermiştir. Bununla birlikte TeV ölçeğinin ötesine geçildiğinde bu modelin parçacık fiziğinin son teorisi olamayacağına inanılır. Günümüzde yapılan parçacık fiziği deney sonuçlarının henüz yeni fizik etkilerine bir kanıt oluşturmadığı göz önünde bulundurulursa, bu etkilerin SM'nin efektif alan teorisi genişletmesinde ortaya çıkması akla yatkındır. Bunlar, daha yüksek boyuttan efektif (etkin) operatörlerden oluşur. Efektif lagranjiyenler temel SM lagranjiyenine eklenecek olan terimlerle elde edilir. Bu terimler SM'de yer almayan parçacıkların bilinen parçacıklarla olan etkileşmelerini içerebilir. Bunun yanında, bu yeni terimler, bilinen parçacıkların SM'de olmayan etkileşmelerinden oluşabilir (anormal etkileşmeler). SM lagranjiyenine kıyasla oldukça küçük katkılar veren bu terimler, bu tür etkileşmelerin neden şimdiye kadar gözlenemediğinin en büyük sebebidir. Ne var ki artan çarpışma enerjileri ve 1şınlık değerleriyle gelecekte bu tür 
etkilerin gözlenmesi öngörülmektedir. Sistemin lagranjiyenini yazarken yerel ayar simetrisi, lepton ve baryon sayısı korunumu ve Lorentz değişmezliği özellikleri gibi temel zorunlulukların, eklenecek olan yeni terimlerde de sağlanması son derece önemlidir. Bu terimlerde dikkat edilecek bir nokta da enerji ölçeğidir. Bu ekstra terimler daha yüksek boyuttan operatörlere sahiptir ve bunun sonucu olarak renormalize edilemezler. Böylelikle de yalnızca sınırlandırılmış bir enerji ölçeğinde geçerli olurlar. Bu enerji ölçeği yeni fiziğin başladığı enerji ölçeğinin altında olmalıdır. Doğal birim sisteminde lagranjiyen [Enerji] ${ }^{4}$ boyutundadır. Dolayısıyla, yüksek boyutlu operatörlerden oluşan etkileşme terimlerinin boyutunu dengelemek için $\Lambda$ enerji ölçeği kullanılmalıdır.

Uyarılmış spin-1/2 nötrino, SM leptonları ve ayar bozonları $\left(V=\gamma, Z, W^{ \pm}\right)$arasındaki etkileşmeler için efektif akım şu şekilde tanımlanmıştır:

$$
J_{1}^{\mu}\left(\frac{1}{2}\right)=\frac{g_{e}}{\Lambda} \bar{u}\left(p, \frac{1}{2}\right) i \sigma^{\mu v} q_{v}\left(1-\gamma_{5}\right) f_{V} u\left(k, \frac{1}{2}\right)
$$

Burada, $\Lambda$ yeni fizik ölçeği; $g_{e}$ elektromanyetik bağlaşım sabiti $\left(g_{e}=\sqrt{4 \pi \alpha}\right) ; k, p$ ve $q$ sırasıyla SM lepton, uyarılmış spin-1/2 nötrino ve ayar bozonunun dörtlü momentumu; $f_{V}$ ayar bozonu $V$ 'ye karşılık gelen yeni elektrozayıf bağlaşım parametresi ve $\gamma^{\mu}$ Dirac matrisi olmak üzere $\sigma^{\mu v}=i\left(\gamma^{\mu} \gamma^{v}-\gamma^{v} \gamma^{\mu}\right) / 2$ şeklinde tanımlanmıştır. Bir uyarılmış nötrino, üç mümkün bozunma genişliğine sahiptir. Bu bozunum modları, $v^{*} \rightarrow v \gamma$ (1şınımsal bozunum), $v^{*} \rightarrow v Z$ (yüksüz zayıf bozunum) ve $v^{*} \rightarrow e W$ (yüklü zayıf bozunum) şeklindedir. Spin-1/2 nötrinonun bozunma genişliği, SM leptonlarının kütleleri ihmal edilerek, şu şekilde bulunur:

$$
\Gamma_{1}\left(l^{*(1 / 2)} \rightarrow l V\right)=\frac{\alpha m^{* 3}}{4 \Lambda^{2}} f_{V}^{2}\left(1-\frac{m_{V}^{2}}{m^{* 2}}\right)^{2}\left(1+\frac{m_{V}^{2}}{2 m^{* 2}}\right)
$$

Burada $f_{\gamma}=\left(f-f^{\prime}\right) / 2, f_{Z}=\left(f \cot \theta_{W}+f^{\prime} \tan \theta_{W}\right) / 2$ ve $f_{W}=f / \sqrt{2} \sin \theta_{W}$ 'dır; $\theta_{W}$ zayıf karışım açısı ve $m_{V}$ ayar bozonunun kütlesidir.

Uyarılmış spin-3/2 nötrino, SM leptonları ve ayar bozonları arasındaki etkileşmeler için iki adet fenomenolojik akım şu şekilde tanımlanmıştır:

$$
\begin{aligned}
& J_{1}^{\mu}\left(\frac{3}{2}\right)=g_{e} \bar{u}^{\mu}\left(p, \frac{3}{2}\right)\left(c_{1 V}-c_{1 A} \gamma_{5}\right) u\left(k, \frac{1}{2}\right) \\
& J_{2}^{\mu}\left(\frac{3}{2}\right)=\frac{g_{e}}{\Lambda} \bar{u}^{\lambda}\left(p, \frac{3}{2}\right) q_{\lambda} \gamma^{\mu}\left(c_{2 V}-c_{2 A} \gamma_{5}\right) u\left(k, \frac{1}{2}\right)
\end{aligned}
$$

Burada $u^{\mu}(p, 3 / 2)$ Rarita-Schwinger vektör spinörünü temsil eder [44]. $\Lambda$ yeni fizik ölçeği; $k, p$ ve $q$ sırasıyla SM lepton, uyarılmış spin-1/2 nötrino ve ayar bozonunun dörtlü momentumudur. $c_{i V}$ ve $c_{i A}$ yeni serbest bağlaşım parametreleridir.

$\mathbf{J}_{1}$ ve $\mathbf{J}_{2}$ akımları dikkate alındığında, uyarılmış spin-3/2 nötrinoların $v^{*} \rightarrow$ vy bozunma modu için bozunma genişliğini veren ifadeler aşağıdaki gibidir:

$$
\begin{aligned}
& \Gamma_{1}\left(v^{*(3 / 2)} \rightarrow v \gamma\right)=\frac{\alpha}{4}\left(c_{1 V}^{\gamma}{ }^{2}+c_{1 A}^{\gamma}{ }^{2}\right) m^{*} \\
& \Gamma_{2}\left(v^{*(3 / 2)} \rightarrow v \gamma\right)=\frac{\alpha}{24}\left(c_{2 V}^{\gamma}{ }^{2}+c_{2 A}^{\gamma}{ }^{2}\right) m^{*}\left(\frac{m^{*}}{\Lambda}\right)^{2}
\end{aligned}
$$
gibidir:

Yüklü $\left(v^{*} \rightarrow e W\right)$ ve yüksüz $\left(v^{*} \rightarrow v Z\right)$ zayıf bozunma modları için bozunma genişlikleri de aşağıdaki

$$
\begin{aligned}
& \Gamma_{1}\left(v^{*(3 / 2)} \rightarrow l V\right)=\frac{\alpha}{48}\left(c_{1 V}^{2}+c_{1 A}^{2}\right) m^{*} \frac{(1-\kappa)^{2}}{\kappa}\left(1+10 \kappa+\kappa^{2}\right) \\
& \Gamma_{2}\left(v^{*(3 / 2)} \rightarrow l V\right)=\frac{\alpha}{48}\left(c_{2 V}^{2}+c_{2 A}^{2}\right) m^{*}\left(\frac{m^{*}}{\Lambda}\right)^{2} \frac{(1-\kappa)^{4}}{\kappa}(1+2 \kappa)
\end{aligned}
$$

Burada $\kappa=\left(m_{V} / m^{*}\right)^{2}, V=Z, W$ ve $l=e, v$ şeklindedir. 
Böylelikle $\mathrm{J}_{1}$ akımı kullanılarak uyarılmış spin-3/2 nötrinoların her bir kütle değerine karşılık elde edilen dallanma oranları (BR) yüzde olarak Tablo 1'de verilmiştir. Parantez içindeki değerler $\mathbf{J}_{2}$ akımı ile elde edilen sonuçları göstermektedir. Burada tüm serbest parametreler $\left(c_{i V}^{\gamma}, c_{i A}^{\gamma}, c_{i V}^{W}, c_{i A}^{W}, c_{i V}^{Z}, c_{i A}^{Z} ; i=1,2\right) 0,5$ alınmıştır. Görüldüğü gibi uyarılmış nötrinonun en büyük geçiş modu $W e$ 'dir.

Tablo 1. Uyarılmış spin-3/2 nötrinoların kütlelerine göre $\mathrm{J}_{1}\left(\mathrm{~J}_{2}\right)$ akımlarıyla elde edilmiş dallanma oranları (BR).

\begin{tabular}{llll}
\hline $\mathbf{m}^{*}(\mathbf{G e V})$ & \%BR $(\mathbf{W}, \mathbf{e})$ & \%BR $\left(\mathbf{Z}, \mathbf{v}_{\mathbf{e}}\right)$ & \%BR $\left(\gamma, \mathbf{v}_{\mathbf{e}}\right)$ \\
\hline 1600 & $55,20(56,10)$ & $43,10(43,60)$ & $1,64(0,29)$ \\
1800 & $55,40(56,20)$ & $43,30(43,60)$ & $1,20(0,23)$ \\
2000 & $55,60(56,20)$ & $43,40(43,60)$ & $1,00(0,18)$ \\
2200 & $55,70(56,20)$ & $43,40(43,60)$ & $0,80(0,15)$ \\
2200 & $55,80(56,20)$ & $43,50(43,70)$ & $0,70(0,13)$ \\
2400 & $55,80(56,20)$ & $43,50(43,70)$ & $0,70(0,13)$ \\
2600 & $55,90(56,20)$ & $43,50(43,70)$ & $0,60(0,11)$ \\
2800 & $55,90(56,20)$ & $43,50(43,70)$ & $0,55(0,09)$ \\
3000 & $56,0(56,20)$ & $43,60(43,70)$ & $0,40(0,08)$ \\
\hline
\end{tabular}

Bununla birlikte farklı iki fenomenolojik akım ( $\mathrm{J}_{1}$ ve $\left.\mathrm{J}_{2}\right)$ için uyarılmış spin-3/2 nötrinoların kütlesine bağlı olarak toplam bozunma genişliğgi Şekil 1'de gösterilmiştir. Burada $\Lambda=m^{*}$ ve tüm serbest bağlaşım parametreleri 0,5 alınmıştır. Beklendiği gibi kütle arttıkça bozunma genişliği değerleri de artmıştır. Ayrıca kütlenin yüksek değerlerine doğru farklı iki akımdan kaynaklı eğriler birbirine yaklaşmaktadır.

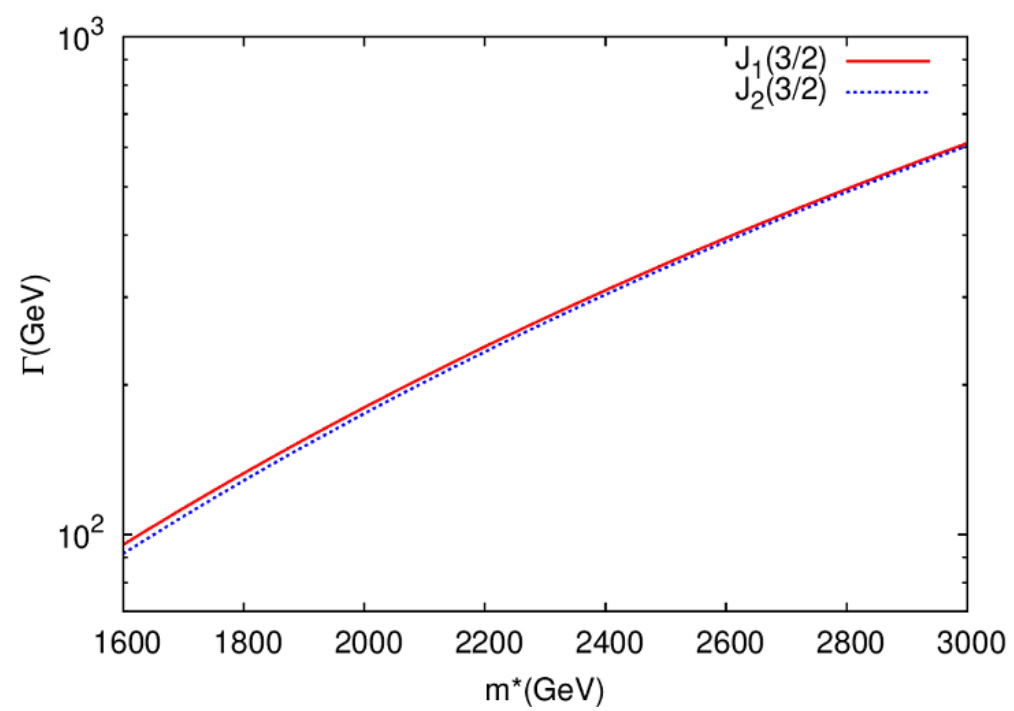

Şekil 1. Uyarılmış spin-3/2 nötrinoların kütlesine bağlı toplam bozunma genişlikleri. 


\section{UYARILMIŞ SPIN-3/2 NÖTRINNOLARIN DOĞRUDAN OLMAYAN ÜRETIMİ}

Bu çalışmada, foton indüklü $\mathrm{e}^{-} \gamma \rightarrow \mathrm{ve}_{\mathrm{e}} \mathrm{W}^{-}$süreci $\mathrm{e}^{-} \mathrm{e}^{+}$çarpışmasındaki bir alt süreci olarak ele alınmış ve tkanalında $v^{*}$ değiş-tokuşu ile uyarılmış spin-3/2 nötrinonun doğrudan olmayan yolla üretimi incelenmiştir. Şekil 2 (a) bu sürecin Feynman diyagramını, (b) ve (c) ise SM ardalan diyagramlarını göstermektedir.

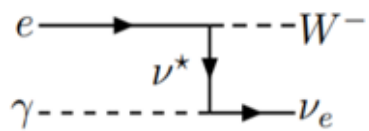

(a)

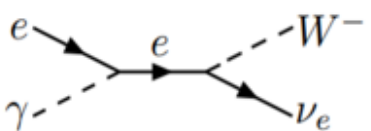

(b)

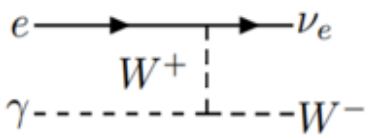

(c)

Şekil 2. $\mathrm{e}^{-} \gamma \rightarrow \mathrm{v}_{\mathrm{e}} \mathrm{W}^{-}$sürecinin Feynman diyagramları. (a) sinyal, (b) ve (c) SM ardalan için.

Lineer çarpıştırıcıda enerjik foton demetlerini üretmek için Compton geri saçılan foton dağılımı kullanılmıştır. Compton geri saçılan fotonların spektrumu şu şekildedir [45, 46]:

$$
f_{y}(x)=\frac{1}{g(y)}\left[1-x+\frac{1}{1-x}-\frac{4}{y(1-x)}+\frac{4 x^{2}}{y^{2}(1-x)^{2}}\right]
$$

Burada $g(y), x, y$ ve $x_{m a k s}$ sırasıyla aşağıdaki gibi tanımlanmıştır:

$$
\begin{aligned}
& g(y)=\left(1-\frac{4}{y}-\frac{8}{y^{2}}\right) \log (y+1)+\frac{1}{2}+\frac{8}{y}-\frac{1}{2(y+1)^{2}} \\
& x=\frac{E_{y}}{E_{e}}, \quad y=\frac{4 E_{0} E_{e}}{M_{e}^{2}}, \quad x_{\text {maks }}=\frac{y}{1+y}
\end{aligned}
$$

Burada $E_{0}$ ve $E_{e}$ sırasıyla, gelen lazer fotonunun enerjisi ve Compton geri saçılmasından önce elektron demetinin başlangıç enerjisidir. $E_{\gamma}$ ise geri saçılan fotonun enerjisidir. $y=4,8$ olduğunda $x$ 'in maksimum değeri yaklaşık olarak 0,83 olur. Buna göre gelen elektron ya da pozitron, enerjisinin \% $\% 3$ 'ünü fotona aktarmış olur. Böylece CLIC, e $e^{+}$sisteminin kütle merkezi enerjisi $\sqrt{s}_{\mathrm{s}}=3000 \mathrm{GeV}$, düşünülen $\mathrm{e}^{-} \gamma$ sistemi için $\sqrt{\mathrm{s}}_{\mathrm{s}}=2733 \mathrm{GeV}$ olacaktır. Çalışmadaki tüm hesaplamalarda CalcHEP yüksek enerji fiziği paket programı kullanılmıştır [47-49].

Tesir kesiti değerleri, $\mathrm{e}^{-} \gamma \rightarrow \mathrm{v}_{\mathrm{e}} \mathrm{W}^{-}$süreci için $\sqrt{\mathrm{s}}_{\mathrm{s}}=2733 \mathrm{GeV}$ alınarak hesaplanmıştır. Buna göre aynı son durumu veren SM ardalan için bu değer $\sigma_{\mathrm{B}}=49,7 \mathrm{pb}$ olarak elde edilmiştir. Bununla birlikte, her bir $v^{*}$ kütle değeri $\left(\mathrm{m}^{*}\right)$ dikkate alınarak aynı kütle merkezi enerjisi için sinyal tesir kesitleri elde edilmiştir. Şekil 3, sinyal tesir kesiti değerlerinin uyarılmış nötrino kütlesine göre grafiğini göstermektedir. Burada üç farklı akım ele alınmıştır; $\mathrm{J}(1 / 2), \mathrm{J}_{1}(3 / 2)$ ve $\mathrm{J}_{2}(3 / 2)$. Ayrıca uyarılmış spin- $1 / 2$ nötrino için $f=-f^{\prime}=1$, uyarılmış spin- $3 / 2$ nötrino için $c_{i V}^{\gamma}=c_{i A}^{\gamma}=c_{i V}^{W}=c_{i A}^{W}=0,5(i=1,2)$ değerleri dikkate alınmıştır. Şekil 3'ten görüldüğü gibi, $\mathbf{J}_{2}(3 / 2)$ akımı için tesir kesiti değerleri, diğer ikisine kıyasla daha yüksektir. 


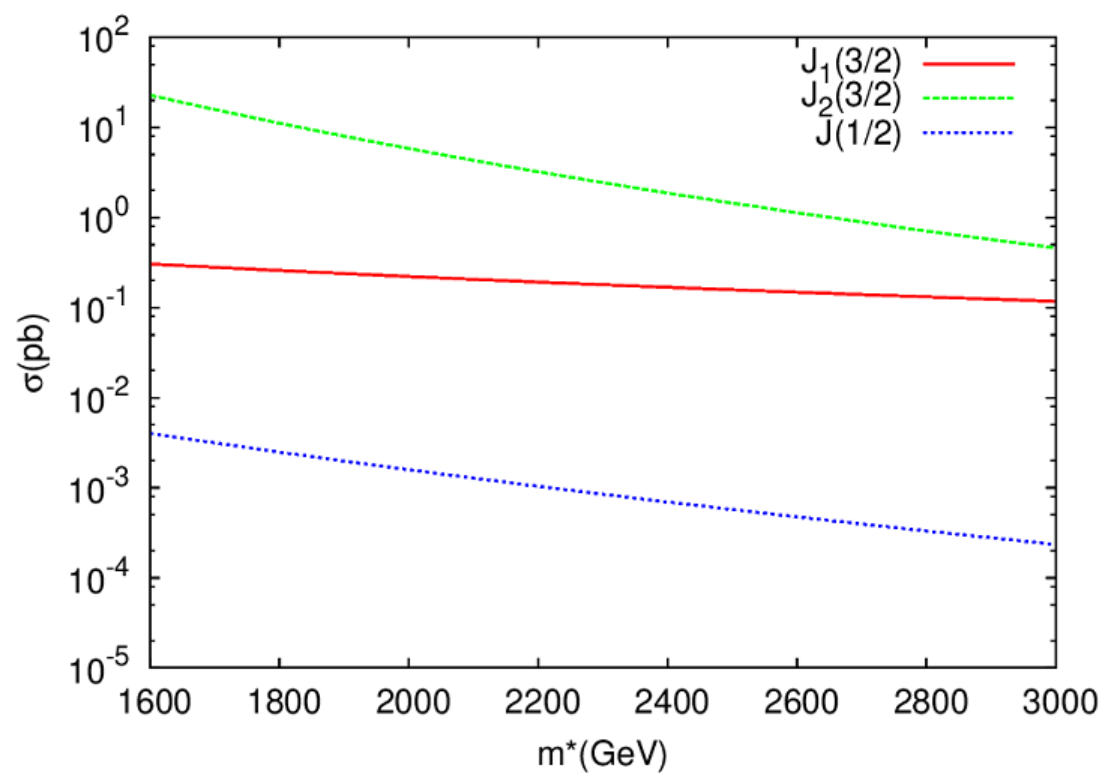

Şekil 3. Uyarılmış nötrino kütlesine göre tesir kesiti grafiği.

Sinyal tesir kesiti değerleri SM ardalan değerinden sapmalar göstermiştir. Bu sapmalara sebep olan etki efektif lagranjiyendeki yeni etkileşme terimlerinden kaynaklanmıştır. Bu etkilerin deneysel gözlenebilirliğinin saptanması için istatistik analiz yöntemleri kullanılabilir. Bu çalışmada serbest bağlaşım parametrelerinin duyarlılık limitlerinin belirlenebilmesi için aşağıdaki gibi tanımlanan istatistik önem (SS, Statistical Significance) yöntemi kullanılmıştır.

$$
S S=\frac{N_{S}}{\sqrt{N_{B}}} ; \quad N_{S}=L_{i n t} \cdot \sigma_{S} \cdot B R \cdot \varepsilon, N_{B}=L_{i n t} \cdot \sigma_{B} \cdot B R \cdot \varepsilon
$$

Burada $N_{S}$ ve $N_{B}$ sırasıyla sinyal ve SM ardalan için olay sayıları; $L_{\text {int }}$, yıllık toplanmış (integre edilmiş) ışınlık; $\sigma_{S}$ ve $\sigma_{B}$ sırasıyla sinyal ve SM ardalan için tesir kesiti; $B R$, dallanma oranı ve $\varepsilon$, verimlilik olarak tanımlanır.

Serbest bağlaşım parametrelerinin ikili korelasyonu sonucu (-1 ve +1 arasında değişen çeşitli değerler) sinyal tesir kesiti değerleri tekrar elde edilmiş ve \%95 güvenirlilik seviyesinde iki-boyutlu kontur grafikleri uyarılmış nötrinonun üç farklı kütle değeri için $(1600,2000$ ve $2400 \mathrm{GeV}$ ) elde edilmiştir (Şekil 4 ve 5). Bunlar iki parametrenin de aynı anda sınırlandırıldığı durumlardır. Bu grafiklerde yalnızca $J_{2}(3 / 2)$ akımı ele alınmış ve $\Lambda$ $=\mathrm{m}^{*}$ eşitliği göz önünde bulundurulmuştur. Örneğin Şekil 4 elde edilirken $c_{2 V}^{W}$ ve $c_{2 A}^{W},-1$ ve +1 arasında değişen pek çok değer alınarak tarama yapılmış ve her an iki değere karşılık gelen tesir kesitleri elde edilmiştir. Bu hesaplama yapılırken $c_{2 V}^{\gamma}=c_{2 A}^{\gamma}=0,05$ alınarak bu parametrelerin etkisi oldukça azaltılmıştır. Benzer biçimde, Şekil 5'te de parametreler için tam tersi durum gerçekleşmiştir. Ancak Şekil 5 'te eğrilerin kapanmamasının nedeni olarak $c_{2 V}^{\gamma}=-c_{2 A}^{\gamma}$ eşitliği gösterilebilir. Bu iki parametre, her ikisinin de aynı, fakat zıt işaretli sayı değerini aldığında tesir kesiti değeri sıfır olmaktadır. Her iki grafikte de, beklendiği gibi, uyarılmış spin-3/2 nötrinonun artan kütle değeriyle tesir kesiti değerleri azaldığından duyarlılık limitleri de kötüleşmektedir; yani, limit değerleri büyümektedir. Ayrıca bu grafikler çizilirken iki parametreyi içeren fit fonksiyonları kullanılmıştır. Bununla birlikte, CLIC için $L_{\text {int }}=5000 \mathrm{fb}^{-1}, B R=0,68$ (W $\rightarrow$ hadronik) alınmış ve $\varepsilon=1$ varsayılmıştır. 


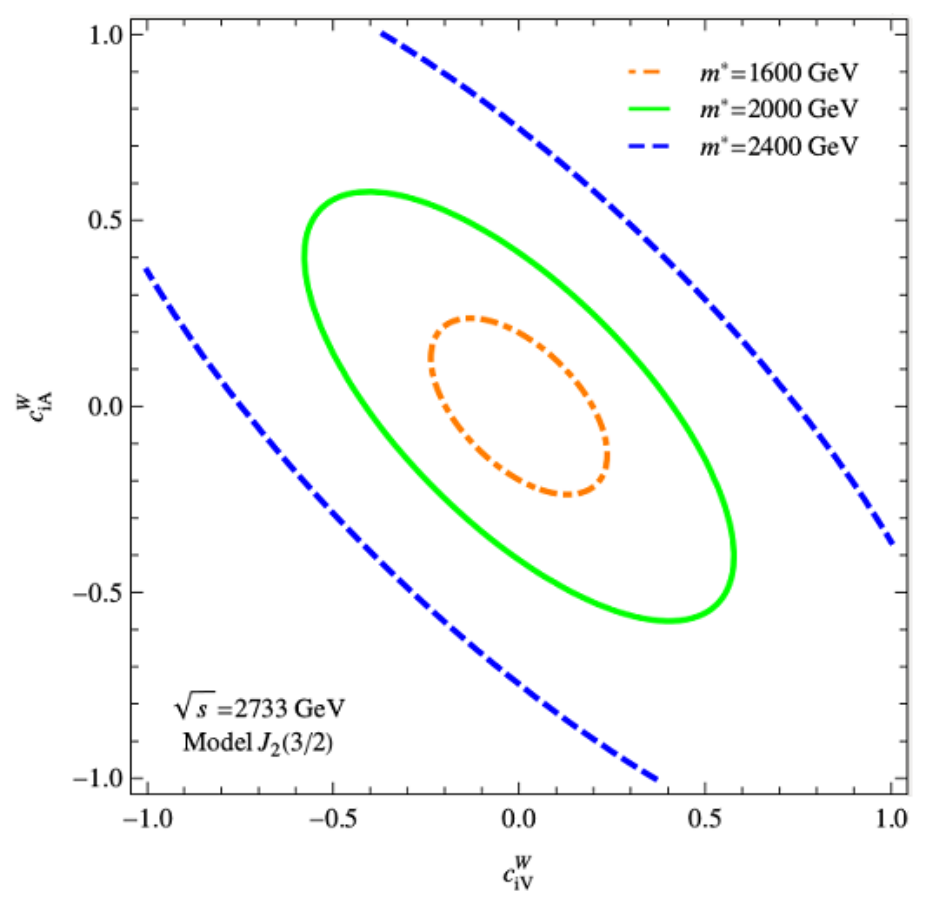

Şekil 4. İki boyutlu kontur grafiği $\left(c_{2 V}^{W}-c_{2 A}^{W}\right)$

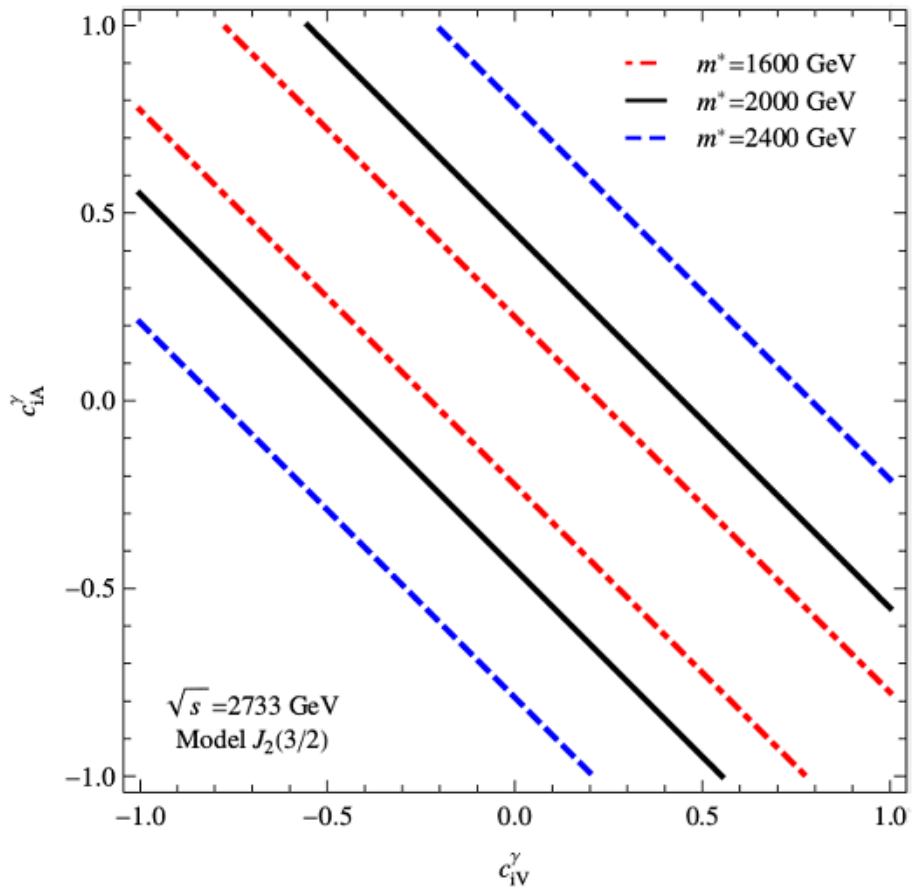

Şekil 5. İki boyutlu kontur grafiği $\left(c_{2 V}^{\gamma}-c_{2 A}^{\gamma}\right)$.

Ayrıca, $c_{i V}^{\gamma}=c_{i A}^{\gamma}=c_{i V}^{W}=c_{i A}^{W}=0,5(i=1,2)$ sabit değerleri dikkate alınarak SS değerleri iki fenomenolojik akım için de hesaplanmıştır (Tablo 2). Burada $\sqrt{\mathrm{s}}=2733 \mathrm{GeV}, \Lambda=\mathrm{m}^{*}, L_{\text {int }}=5000 \mathrm{fb}^{-1}$ ve $B R=$ 0,68 alınmıştır. SS $\geq 3$ kriteri parçacığın $\% 95$ güvenirlilik seviyesinde gözleneceğine işarettir. 
Tablo 2. $\mathrm{J}_{1}$ ve $\mathrm{J}_{2}$ akımı ve uyarılmış spin-3/2 nötrinoların kütlelerine göre SS değerleri.

\begin{tabular}{cll}
\hline $\mathbf{m}^{*}(\mathbf{G e V})$ & $\mathbf{S S}\left(\mathbf{J}_{\mathbf{1}}\right)$ & $\mathbf{S S}\left(\mathbf{J}_{\mathbf{2}}\right)$ \\
\hline 1600 & 79,9 & 5978,6 \\
1700 & 73,2 & 4101,7 \\
1800 & 67,4 & 2879,4 \\
1900 & 62,3 & 2055,6 \\
2000 & 57,7 & 1493,7 \\
2100 & 53,7 & 1102,9 \\
2200 & 50,1 & 825,8 \\
2300 & 46,8 & 626,5 \\
2400 & 43,8 & 481,1 \\
2500 & 41,1 & 372,9 \\
2600 & 38,7 & 292,2 \\
2700 & 36,4 & 231,1 \\
2800 & 34,4 & 184,4 \\
2900 & 32,6 & 148,2 \\
3000 & 30,8 & 120,2 \\
\hline
\end{tabular}

\section{III.SONUÇLAR}

$\mathrm{Bu}$ çalışmada, e çarpışmasındaki $v_{\mathrm{e}} \mathrm{W}$ üretiminde uyarılmış spin-3/2 nötrino sinyalinin t-kanalında doğrudan olmayan üretimi araştırılmıştır. Bunun için Compton geri saçılan foton dağılımı kullanılmıştır. Dolayısıyla tüm hesaplamalarda $2733 \mathrm{GeV}$ kütle merkezi enerjisi ve $5000 \mathrm{fb}^{-1}$ yıllık 1şınlık değeri dikkate alınmıştır. Bunun yanında elde edilen tüm sonuçlar için yeni fizik enerji ölçeği, uyarılmış nötrino kütlesine eşit alınmıştır. $\mathrm{Bu}$ hesaplamalarda iki farklı fenomenolojik akım $\left(\mathrm{J}_{1}(3 / 2)\right.$ ve $\left.J_{2}(3 / 2)\right)$ kullanılmıştır. Uyarılmış nötrinoların değişken kütlelerine karşın serbest bağlaşım parametreleri çalışmanın büyük bir bölümünde sabit ve 0,5 değerinde alınmıştır. Çünkü bu parametrelerin teorik bir öngörüsü yoktur; efektif akımlar bu parametre değerlerini tahmin edememektedir. Çalışma boyunca bu akımlar arasında olabilecek girişimler de göz önünde bulundurulmamıştır. SS $\geq 3$ kriteri dikkate alındığında, 3000GeV kütle değeri de dâhil, uyarılmış nötrinoların bu kütle değerlerine kadar \%95 güvenirlilik seviyesinde doğrudan olmayan üretimi her iki akım için de gözlenebilir. Bunun yanında, bu çalışmada 1600, 2000 ve $2400 \mathrm{GeV}$ uyarılmış nötrino kütle değerleri için serbest bağlaşım parametrelerinin aynı anda sınırlandırıldığı iki-boyutlu kontur grafikleri $\mathbf{J}_{2}$ akımı için elde edilmiştir. Buna göre, daha küçük kütle değerinde daha çok bölgenin sınırlandırıldığı gözlenmiştir.

Proton-proton çarpışmalarında, çarpışma sonrası protonlar partonlarına ayrışırlar. Bunun sonucunda çarpışmanın ardalanı büyük ölçüde parton kalıntılarından oluşur. Böylece bu çarpışmalar temiz bir çevresel ortam sağlayamazlar. Genel olarak lepton çarpıştırıcıları, hadron çarpıştırıcılarına nazaran daha temiz bir çevresel ortama; yani, daha temiz çarpışma ardalanına sahiptir. Bu yönüyle de bu çarpışmalar yeni bir parçacığın araştırılmasında son derece önemlidir. Böylece CLIC gibi yüksek 1şınlık ve yüksek kütle merkezi enerjisine sahip gelecek nesil lepton çarpıştırıcısı projeleri bu tür araştırmaları yapmak için iyi bir firsat sunar. Lepton çarpıştırıcılarında normal çalışma modunun yanı sıra foton indüklü reaksiyonlar kendiliğinden ortaya çıkacaktır. Gerek duyulan donanımların kullanılması, bu reaksiyonların, çarpıştırıcının ana modundan ayırt edilebilmesini sağlar. Çalışma sonuçları, CLIC e $\gamma$ modunun uyarılmış spin-3/2 nötrino sinyalini araştırma potansiyeline sahip olduğunu göstermiştir.

\section{KAYNAKLAR}

[1] Terazawa, H., Chikashige, Y., \& Akama, K. (1977). Unified Model of the Nambu-Jona-Lasinio Type for All Elementary-Particle Forces. Physical Review D, 15 (2), 480-487.

[2] Ne’eman, Y. (1979). Primitive Particle Model. Physics Letters B, 82 (1), 69-70.

[3] Terezawa, H., Yasu`e, M., Akama, K., \& Hayashi, M. (1982). Observable Effects of the Possible Substructure of Leptons and Quarks. Physics Letters B, 112 (4-5), 387-392.

[4] Renard, F. M. (1983). Excited Quarks and New Hadronic States. Il Nuovo Cimento A, 77 (1), 1-20. 
[5] Eichten, E. J., Lane, K. D., \& Peskin, M. E. (1983). New Tests for Quark and Lepton Substructure. Physical Review Letters, 50 (11), 811-814.

[6] de R'ujula, A., Maiani, L., \& Petronzio, R. (1984). Search for Excited Quarks. Physics Letters B, 140 (3-4), 253-258.

[7] Kühn, J., \& Zerwas, P.M. (1984). Excited Quarks and Leptons. Physics Letters B, 147 (1-3), 189-196.

[8] Çakır, O., \& Ozansoy, A. (2009). Single Production of Excited Spin-3/2 Neutrinos at Linear Colliders. Physical Review D, 79 (5).

[9] Hagiwara, K., Komamiya, S., \& Zeppenfeld, D. (1985). Excited Lepton Production at LEP and HERA. Zeitschrift für Physik C Particles and Fields, 29 (1), 115-122.

[10] Belyaev, A., Boos, E., \& Pukhov, A. (1992). Study of Excited Neutrino Production in $\mathrm{e}^{+} \mathrm{e}^{-}, \gamma \mathrm{e}$ and $\gamma \gamma$ Collisions at TeV Energies. Physics Letters B, 296 (3-4), 452 - 457.

[11] Ginzburg, I. F., \& Ivanov, D. Y. (1992). Excited Leptons and Quarks at $\gamma \gamma / \gamma$ e Colliders. Physics Letters B, $276(1-2), 214-218$.

[12] Boudjema, F., Djouadi, A., \& Kneur, J. L. (1993). Excited Fermions at $\mathrm{e}^{+} \mathrm{e}^{-}$and ep Colliders. Zeitschrift fur Physik C Particles and Fields, 57 (3), 425-449.

[13] 'Eboli, O. J. P., Gregores, E.M., Montero, J. C., Novaes, S. F., \& Spehler, D. (1996). Excited Leptonic States in Polarized e- $\gamma$ and e+e-Collisions. Physical Review D, 53 (3), $1253-1263$.

[14] 'Eboli, O. J., Lietti, S. M., \& Mathews, P. (2002). Excited Leptons at The CERN Large Hadron Collider. Physical Review D, 65 (7).

[15] Boos, E., Vologdin, A., Toback, D. \& Gaspard, J. (2002). Prospects of Searching for Excited Leptons During Run II of the Fermilab Tevatron. Physical Review D, 66 (1).

[16] Kirca, Z., Cakir, O., \& Aydin, Z. Z. (2003). Production of Excited Electrons at TESLA and CLIC Based e Gamma Colliders. Acta Physica Polonica B, 34 (8).

[17] Cakir, O., Leroy, C., Mehdiyev, R., \& Belyaev, A. (2004). Production and Decay of Excited Electrons at the LHC. The European Physical Journal C, 32 (supplement 2), 1-17.

[18] Cakir, O., Y1lmaz, A., \& Sultansoy, S. (2004). Single Production of Excited Electrons at Future $\mathrm{e}^{+} \mathrm{e}^{-}$, ep and pp Colliders. Physical Review D, 70 (7).

[19] Cakir, O., Türk Cakir, İ., \&Kırca, Z. (2004). Single Production of Excited Neutrinos at Future $\mathrm{e}^{+} \mathrm{e}^{-}$, ep and pp Colliders. Physical Review D, 70 (7).

[20] Belyaev, A., Leroy, C., \& Mehdiyev, R. (2005). Production of Excited Neutrinos at the LHC. The European Physical Journal C, 41 (supplement 2), 1-10.

[21] Acosta, D., Adelman, J., Affolder T. et al. (2005). Search for Excited and Exotic Electrons in the e $\gamma$ Decay Channel in $p p$ Collisions at $\sqrt{ } s=1.96 \mathrm{TeV}$. Physical Review Letters, 94 (ID 101802).

[22] Abazo, W. M., Bloom, K., \& Snow, G. R. (2008). Search for Excited Electrons in $p p$ Collisions at $\sqrt{ } s=1.96$ TeV. Physical Review D, 77 (9).

[23] Inan, S. C. (2010). Exclusive Excited Leptons Search in Two Lepton Final States at the CERN LHC. Physical Review D, 81 (11). 
[24] Ozansoy A. \& Billur, A. A. (2012). Search for Excited Electrons Through $\gamma \gamma$ Scattering. Physical Review D, 86 (5).

[25] Köksal, M. (2014). Analysis of Excited Neutrinos at the CLIC. International Journal of Modern Physics A, 29 (24).

[26] Caliskan, A., Kara, S. O., \& Ozansoy, A. (2017). Excited Muon Searches at the FCC-Based Muon-Hadron Colliders. Advances in High Energy Physics, 2017 (1540243).

[27] Caliskan, A., (2017). Excited Neutrino Search Potential of the FCC-Based Electron-Hadron Colliders. Advances in High Energy Physics, 2017 (4726050).

[28] Caliskan, A., \& Kara, S. O. (2018). Single Production of the Excited Electrons in the Future FCC-Based Lepton-Hadron Colliders. International Journal of Modern Physics A, 33 (24).

[29] Caliskan, A., (2019). Search for Excited Muons at the Future SPPC-based Muon-Proton Colliders. Acta Physica Polonica B, 50, 1409-1422.

[30] Choudhury, S. R., Ellis, R. G., \& Joshi, G. C. (1985). Limits on Excited Spin-3/2 Leptons. Physical Review D, 31 (9), 2390-2392.

[31] Spehler, D., 'Eboli, O. J., Marques, G. C., Novaes, S. F. \& Natale, A. A. (1987). Looking for Spin-3/2 Leptons in Hadronic Collisions. Physical Review D, 36 (5), 1358 - 1362.

[32] Almeida, F., Martins Simoes, J., \& Ramalho, A. (1993). Lepton Production at HERA. Nuclear Physics B, 397 (3), 502-514.

[33] Almeida Jr., F. M. L., Lopes, J. H. Martins Simoes, J. A. \& Ramalho, A. J. (1996). Production and Decay of Single Heavy Spin-3/2 Leptons in High Energy Electron-Positron Collisions. Physical Review D, 53 (7), $3555-3558$.

[34] Cakir, O., \& Ozansoy, A. (2008). Search for Excited Spin-3/2 and Spin-1/2 Leptons at Linear Colliders. Physical Review D, 77 (3).

[35] Ozansoy, A., Arı, V., \& Cetinkaya, V. (2016). Search for Excited Spin-3/2 Neutrinos at LHeC, Advances in High Energy Physics, 2016, (ID 1739027).

[36] Achard, P., Adriani, O., Aguilar-Benitez, M. et al. (2003). Search for Excited Leptons at LEP. Physics Letters $B, 568$ (1-2), $23-34$.

[37] Aaron, F. D. et al. (2008). A Search for Excited Neutrinos in $\mathrm{e}^{-} \mathrm{p}$ Collisions at HERA. Physics Letters B, 663 (5), 382-389.

[38] Aad, G., Abbott, B., Abdallah, J. et al. (2015). Search for New Phenomena in Events with Three or More Charged Leptons in $p p$ Collisions at $\sqrt{ } s=8 \mathrm{TeV}$ with the ATLAS Detector. Journal of High Energy Physics, 2015 (138).

[39] Accomando, E. et al. (2004). Physics at the CLIC Multi-TeV Linear Collider. arXiv: hep-ph/0412251 (hepph), CERN-2004-005.

[40] Dannheim, D., Lebrun, P., Linssen, L., Schulte, D., Simon, F., Stapnes, S., Toge, N., Weerts, H., \& Wells, J. (2012). CLIC e+e- Linear Collider Studies. arXiv: 1208.1402 (hep-ex).

[41] Abramowicz, H, vd. (2013). Physics at the CLIC e e $e^{-}$Linear Collider -- Input to the Snow Mass Process 2013. arXiv:1307.5288 (hep-ex). 
[42] CLIC and CLICdp Collaborations. (2018). The Compact Linear Collider (CLIC) - 2018 Summary Report, CERN Yellow Rep. Monogr. 1802, 1-98.

[43] de Blas, J. et al. (2019). The CLIC Potential for New Physics arXiv:1812.02093v2 (hep-ph).

[44] Rarita, W., \& Schwinger, J. S. (1941). On a Theory of Particles with Half Integral Spin. Physical Review, 60 (1).

[45] Ginzburg, I. F., et al. (1984). Colliding $\gamma e$ and $\gamma \gamma$ Beams Based on Single-Pass $\mathrm{e}^{+} \mathrm{e}^{-}$Accelerators II. Polarization Effects, Monochromatization Improvement. Nuclear Instruments and Methods in Physics Research 219 (5).

[46] Telnov, V. I. (1990). Problems in Obtaining $\gamma \gamma$ and $\gamma$ e Colliding Beams at Linear Colliders. Nuclear Instruments and Methods in Physics Research, 294 (72) (Sect. A).

[47] Pukhov, A., Boos, E., Dubinin, M., et al., (2000). CompHEP-a Package for Evaluation of Feynman Diagrams and Integration over Multi-Particle Phase Space. User's Manual for Version 33. https://arxiv.org/abs/hep$\mathrm{ph} / 9908288 \mathrm{v} 2,08.11 .2019$

[48] Pukhov, A. (2009). CalcHEP 2.3: MSSM, Structure Functions, Event Generation, Batchs, and Generation of Matrix Elements for Other Packages. https://arxiv.org/abs/hep-ph/0412191v2, 08.11.2019

[49] Belyaev, A., Christensen, N. D., \& Pukhov, A. (2013). CalcHEP 3.4 for Collider Physics Within and Beyond the Standard Model,” Computer Physics Communications, 184 (7), 1729 - 1769. 